\title{
TRABAJO, JÓVENES E IDENTIDAD EN UN CONTEXTO DE CRISIS DE LOS REFERENTES MODERNOS: UNA APROXIMACIÓN A LA FIGURA DEL EMPRENDEDOR
}

\section{Work, youth and identity in a context of crisis of modern concerning: an approach to the entrepreneur}

\author{
Diego Carbajo Padilla*; Elsa Santamaría López** \\ * Universidad del País Vasco / EuskalHerrikoUnibertsitatea; ** Universidad Oberta de Catalunya \\ (UOC) \\ *diego.carbajo@ehu.eus; **esantamarial@uoc.edu
}

Palabras clave

Modernidad

Trabajo

Individuo

Emprendimiento

Juventud

\section{Keywords}

Modernity

Work

Individual

Entrepreneurship

Youth

\section{$\underline{\text { Resumen }}$}

Junto con cuestiones como el nacionalismo, las identidades o la secularización, uno los ejes transversales en la obra de Alfonso Pérez-Agote es el de los procesos de desinstitucionalización. Este artículo toma este eje como potente herramienta teórica para estudiar las transformaciones del mundo del trabajo y de la juventud y tiene como objetivo, a través de su análisis y discusión, actualizarlo y situarlo en una de las cuestiones que ha adquirido fuerza en los últimos años: el emprendimiento. Queremos evidenciar con ello que las contribuciones de Alfonso Pérez-Agote permiten desarrollar líneas de reflexión muy productivas para encarar sociológicamente cambios que, a día de hoy, se están produciendo en lo laboral y que también lo transcienden.

\begin{abstract}
Together with issues like nationalism, identity or secularization, the deinstitutionalization process is one of the main transversal axes in the work of Alfonso Pérez-Agote. This article takes recourse to this powerful theoretical tool to address the transformations that are taking place with in the labour market and the youth. By analysing and discussing it, our aim is to update and place it around an issue that has gained relevance in recent years: the entrepreneurship. There by we want to stress that Alfonso Pérez-Agote's contributions enable to develop strong lines of sociological thoughts to deal with the changes that are taking place not only in the labour market, but beyond.
\end{abstract}

Carbajo, D., Santamaría, E., 2015, “Trabajo, jóvenes e identidad en un contexto de crisis de los referentes modernos: una aproximación a la figura del emprendedor", en Papeles del CEIC, vol. 2015/3, n' 143, CEIC (Centro de Estudios sobre la Identidad Colectiva), Universidad del País Vasco, http://dx.doi.org/10.1387/pceic.14626 


\section{INTRODUCCIÓN}

Si en algo se caracteriza la obra del Catedrático de Sociología Alfonso Pérez-Agote es la mixtura de abordajes teóricos y empíricos y el calado de su pensamiento en múltiples ámbitos de estudio. En este artículo recogemos algunas de sus reflexiones teóricas más agudas y sugerentes para aproximarnos a un objeto y un sujeto, al trabajo y a la juventud, que han sido abordados en varias de sus investigaciones empíricas. Los cambios que actualmente se reflejan en esas dos categorías nos remiten, de vuelta, al propio ejercicio de reflexión teórica sobre esas conceptualizaciones. Con este sencillo ejercicio queremos dejar constancia de uno de los principios que él siempre ha trasmitido, que es estructurante en su obra, y que a día de hoy conviene seguir teniendo presente aunque parezca obvio: Que la teoría es indispensable para realizar un trabajo empírico serio y que la teoría social no se sostiene sin observaciones empíricas.

Desde esta premisa, el recorrido que planteamos parte de la revisión de algunas de sus contribuciones en torno a la modernidad, el Estado, el individuo y la institución del trabajo y termina profundizando en algunas transformaciones contemporáneas que tienen mucho que ver con la juventud y el trabajo. Con este objetivo revisamos algunos de sus artículos para profundizar primero en el relato de la crisis de la modernidad. Seguidamente, abordamos aquellas investigaciones que ha dirigido y en las que la crisis de la institución social del trabajo y la juventud son centrales. Por último, partiendo de sendas bases teóricas y empíricas, elaboramos una serie de reflexiones que se derivan principalmente de nuestros trabajos más recientes en torno al empleo, la juventud y lo que se viene conociendo como emprendimiento.

Con este breve texto - que ha de ser leído, más que como una revisión exhaustiva de sus trabajos, como una invitación o un aperitivo templado para todo el banquete intelectual y académico que supone la obra de Alfonso Pérez-Agote-, queremos mostrar la pertinencia que tiene su pensamiento para seguir reflexionando sobre los actuales cambios sociales. 


\section{El RELATO DE CRISIS DE LA MODERNIDAD, DEL Estado y DEL INDIVIDUO}

La idea central del relato que Pérez-Agote elabora en los textos que hemos trabajado puede resumirse en que actualmente el Estado no es capaz de condensar la totalidad de la vida social y ello nos exige como sociólogos un redoblado esfuerzo teórico. Tal y como argumenta en diferentes obras y artículos, a lo largo del Antiguo Régimen el Estado Nacional se fue gestando como un centro unificador y totalizador sobre un territorio, como una operación política que fundaba la capacidad de acción de un grupo y la capacidad de pensarse a si mismo, en la que posteriormente se produjo una separación entre esfera política y sociedad civil. Articulada respecto a la ecuación entre Estado y Mercado, la emergencia de la burguesía estableció esa separación entre esfera política y sociedad civil haciendo que, protegido por la ley y Estado de derecho, el individuo adquiriese preeminencia sobre el grupo y se convirtiese en una figura central de la modernidad' (Pérez-Agote, 2000: 444).

Sin embargo, a mediados del siglo XX y a medida que la modernidad se aceleraba, el principio expansivo del mercado desbordó la lógica territorial y local del Estado haciendo que esta forma de comprensión basada en el par Estado-Mercado se fuera resquebrajando (Ibid., 2002: 508). El Estado fue perdiendo la capacidad de contención de las propias lógicas del mercado y también de las lógicas sociales que tenían lugar dentro de sí y esa crisis del Estado Nacional, como crisis de la modernidad, se declinó en los individuos como quiebra - parcial- de sus estructuras de plausibilidad. Define así la mundialización como época anómica caracterizada por un menor grado de estructuración de la realidad social. Brevemente, los desplazamientos conceptuales que se han vuelto centrales para

\footnotetext{
${ }^{1}$ Y nos referimos con esto también a la propia representación sociológica de individuo. En uno de sus artículos Pérez-Agote (1996) remarca la historicidad de la ciencia en la que no hay ni universalidad ni transhistoricidad ni del referente empírico ni del concepto que usamos para analizarlo. Da cuenta así de que uno de los problemas teóricos básicos durante la modernidad es que las dicotomías básicas de individuo y sociedad se han pensado como conceptos transhistóricos. Si bien la estrategia de la sociología para consolidarse como disciplina académica pasó por el recurso a la noción de individuo desarrollada en la psicología y le resultó útil en su institucionalización, a la larga se ha revelado muy problemática. La crisis de la sociedad puso en entredicho la existencia del individuo y ello, por fuerza, puso en crisis esa representación analítica. En todo caso, y aunque el autor nos recuerda que la mayoría de las veces es banal decir que una forma social está en crisis, subraya que la sociología ha aceptado mucho más fácilmente la crisis del Estado y de la sociedad que la crisis del individuo. Entraremos en esta cuestión más adelante.
} 
comprenderla serían el paso desde mecanismos de asimilación a la diferenciación cultural y el paso de la lógica del centro-periferia a la interconexión y la lógica en red (Ibid., 1999: 68 y ss.).

En lo que refiere al mencionado concepto de anomia, apoyado en autores como Merton y Parsons, Pérez-Agote lo define como una dislocación entre la dimensión simbólico-territorial en la que vive el actor social y la emergencia de una nueva realidad y estructura social que no obedeciendo a sus marcos de sentido, pierde significación para él. Con otras palabras, es aquí donde identifica una pérdida de la capacidad articuladora del Estado nacional debido a la complejización de las lógicas supranacionales e infranacionales, en las que, como correlato empírico de sus reflexiones, la Unión Europea viene a suponer un ámbito de toma de decisiones lejanas e incompresibles para un actor territorializado que desconoce sus reglas (lbid., 1999).

Brevemente y para lo que nos ocupa, lo que conocemos hoy como el Estado de Bienestar funcionó durante algunas décadas del siglo $X X$, en algunos paises occidentales - y casi excepcionalmente (Ibid., 2002: 523)-, como la forma principal de gestionar colectivamente esos procesos de conflicto, crisis y anomia. La progresiva merma de sus márgenes de maniobra y la erosión de su capacidad de gestión de la crisis de la modernidad ha desembocado en una quiebra de sus mecanismos de legitimación democrática (Ibid., 2000: 451). En este orden de cosas ya desde finales del siglo pasado, el Estado, en tanto que mecanismo central de integración del individuo en la sociedad, evidencia una incapacidad de transformar el crecimiento económico en crecimiento del nivel de empleo y ni siquiera en el mantenimiento de la calidad y estabilidad de este. El cambio básico que supone esta mutación puede resumirse en que el Estado pasa a descargar sobre los individuos muchas de las funciones que anteriormente cumplió. Con todo, este cambio no supone tanto la desaparición del Estado si no una alteración en sus funciones en las que las lógicas que vienen de la modernidad coexisten e interactúan con las que nacen y se despliegan en la contemporaneidad (Ibid., 1999: 61). El pensamiento de Pérez-Agote nos sitúa entonces en el ambivalente quicio de dos épocas que coexisten y entran en conflicto: entre la modernidad y la globalidad (Ibid., 2002: 519 y ss.).

Dentro del carácter anómico de esos procesos destaca la desarticulación de las fuentes de identidad y de sentido del individuo y llama la atención sobre la importancia de la dimensión simbólica de la diferenciación social. 
A saber, la forma en la que los individuos se representan la realidad social en la que viven como unidad o como totalidad (Ibid., 1996: 29). Desde esa posición, las preguntas que trata de responder pueden sintetizarse en: ¿qué pasa con el individuo cuando ese Estado entra en crisis?, ¿cómo ha llegado nuestra cultura a atribuir tanta importancia al individuo? (Ibid., 2000: 454)

Varios de sus artículos vienen a señalar que actualmente, cada persona estaría inscrita en diferentes esferas de la vida social y que cada esfera, siendo autónoma en el plano simbólico en relación a las otras, habría mellado las grandes construcciones simbólicas haciendo que la elaboración del sentido de los sujetos pase al dominio privado o incluso subjetivo (Ibid., 2000: 454). En otras palabras, el individuo tendría que construir, en el interior de su mundo privado, su propia creencia que, en última instancia, sería un sincretismo elaborado de diferentes materiales resultantes de la explosión en el ámbito público, de las codificaciones religiosas y políticas, y de otros elementos provenientes de esferas como la ciencia (Ibid., 1996: 28). Recurriendo a la profecía weberiana del politeísmo de los valores y conceptualizando este proceso como la segmentación del individuo, Pérez-Agote señala que éste también se vería impelido a buscar la pequeña comunidad para intentar objetivar sus creencias a través de la intersubjetividad y la socialidad (Ibid., 2002). En suma, los individuos estarian obligados a manejarse dentro de diferentes planos de sentido y lógicas de acción que entrarían en contradicción recurrentemente ${ }^{2}$. Si bien se subraya que lo político escapa a los marcos de sentido del actor social, también aparecen "nuevas" lógicas de acción y atribución de significado cuando se detecta que la socialidad, la identidad y las instituciones que fueron cooptadas por el Estado - familia, gremio, vecindad, etc.- parecen recuperar un sentido propio (Pérez-Agote, 2000: 452 y ss.).

\footnotetext{
${ }^{2}$ Como conclusión heurística subraya que no parece que realizar el análisis de la realidad contemporánea desde posiciones teóricas, cerradas y totales sea una buena estrategia sociológica. Señala así que la teoría de sistemas, por su carácter cerrado y finalista muestra muchas dificultades para entender los cambios sociales de grados de estructuración bajos. Y con ello nos recuerda que el error teórico del marxismo y del funcionalismo del siglo $X X$ consistió en reducir las dimensiones sociales y de socialidad a meras variables secundarias olvidando que la acción social puede tener sentido por sí misma. Es decir, que en la acción racional también intervienen elementos "no-racionales" como la cultura, la tradición o los afectos y que ellos no necesariamente obedecen siempre a la acción racional con arreglo a fines. En pocas palabras nos sitúa en un escenario donde los comportamientos sociales desbordan constantemente la moderna dicotomía analítica racional-irracional (Ibid., 2002:523).
} 
Y resulta imprescindible subrayar aquí la relación que Pérez-Agote establece entre la práctica teórica de la sociología y las crisis de la modernidad, del Estado y del individuo a las que nos hemos referido. $\mathrm{Si}$ durante la modernidad el núcleo central y los garantes de la misma han sido los adultos varones profesionales en los que su periferia vicaria estaba compuesta por las mujeres, los jóvenes y "algunos no profesionales"(Ibid., 1996: 29), hoy en día, la aparición de nuevas formas de combinación de los elementos constitutivos de la edad social certificarian la inadecuación de las divisiones sociales y analíticas que se corresponden tanto con la edad biológica, con las configuraciones familiares y con la situación laboral. En resumen, en la medida que las definiciones producidas por ese centro ya no sirven como mecanismo de integración para todos, esa posición social central del individuo se desvanece (Ibid., 1996: 30). La pregunta que PérezAgote lanza ante este tipo de procesos es si no habría "que rastrear la significación de fenómenos como la anomia, en sus diferentes variantes analíticas, a través de una revisión de la noción, tan moderna, como la de individuo" (Ibid., 2002: 518).

\section{LA DESINSTITUCIONALIZACIÓN DEL TRABAJO A TRAVÉS DE LA JUVENTUD}

En las investigaciones empíricas que Pérez-Agote dirigió en la primera década del 2000 se pueden apreciar muchas de las líneas de reflexión que dan forma al marco general que hemos descrito en el punto anterior. En gran parte de estas investigaciones se trabajan cuestiones que giran en torno a las transformaciones de diferentes dimensiones laborales y de la identidad, la erosión de los entramados institucionales provistos por el Estado y la Administración vasca, las formas de consumo, la socialidad, la emancipación residencial, etc. Adoptando una perspectiva fenomenológica en la que el conocimiento de las percepciones y las definiciones que los individuos realizan de sus situaciones y posiciones sociales resultan claves para comprender su comportamiento, en estos trabajos se aborda a la juventud vasca como uno de los colectivos que más directa y tempranamente han recibido los cambios, en especial en el mundo del trabajo, pero también como agente del cambio al encajarlos (Pérez-Agote et al., 2005a: 12).

Entre las tendencias que en el momento de realización de estos trabajos eran emergentes y que se han reforzado en la actualidad, destaca primeramente una generalizada valoración negativa del mercado de 
trabajo, pese a los indicadores laborales positivos, y sobre todo entre los jóvenes, que se encontraban con serios obstáculos para su inserción laboral (Pérez-Agote et al, 2001b). Por un lado, el "carácter endémico de la crisis del mercado de trabajo y del empleo" y el "continuum precariedadestabilidad" hacian que la relevancia del trabajo como valor y como deber moral central en la vida disminuyera en los más jóvenes. Sin embargo, al mismo tiempo que la institución social del trabajo se erosionaba por la importancia que parecían adquirir otras actividades, la densidad cultural del trabajo remunerado como mecanismo de integración social se mantenía en un lugar central. La preferencia por el trabajo asalariado y la preocupación social que produce el desempleo serían los marcadores de esta centralidad (Ibid., 2005a: 85 y 89).

Ambas lógicas, aparentemente contrapuestas, venían a señalar un cambio operado en la cultura del trabajo y concretado en la noción de anomia como desajuste entre medios y fines. Así, estos trabajos vienen a dar cuenta de un incipiente choque entre lo prometido por la estructura simbólica y lo obtenido en el mercado de trabajo (Pérez-Agote y Santamaría, 2008: 69 y ss.). Detectan que la inadecuación entre la educación recibida y el posterior trabajo crecía en las personas jóvenes y que la dedicación individual y el esfuerzo familiar realizado no permitían ya entonces salir del bucle más-formación-más-precariedad-más-frustración, e incluso que lo reforzaba (Santamaría, 2011). En sus términos, estos trabajos identifican el incipiente cuestionamiento de la "ética ascética secularizada" según la cual el futuro profesional no es incierto y que depende de lo que se haga hoy en términos de esfuerzo, sacrificio y formación (Pérez-Agote et al., 2005a: 67 y 68).

La cronificación de las condiciones laborales precarias y la escasez de puestos de trabajo hasta entonces normales o típicos reforzaban en los más jóvenes dos tendencias que se han asentado y acrecentado con posterioridad y sobre todo, con la actual crisis económica que arrastramos desde el año 2008. Por un lado, la tendencia que apunta hacia la resignificación de la estabilidad y, por otro, la que gira en torno al autoempleo. En lo que refiere a la primera tendencia, el que la búsqueda de la estabilidad sea un elemento importante para los jóvenes se interpreta como un indicador de la interiorización de las dificultades para encontrar un puesto de trabajo en el mercado laboral. En lo que refiere a la segunda, indica que "la necesidad sentida por muchos de encontrar alguna fórmula de auto-empleo, han borrado la neta frontera que separaba la empresa del 
trabajo asalariado, así como aquella que separaba a los asalariados de los independientes o autónomos" (Pérez-Agote et al. 2005a: 9).

Esta reflexión resulta crucial porque aunque establece matices respecto a que la cultura empresarial estuviese demasiado extendida y que en todo caso se declinaba en términos personales, vocacionales y de independencia, es un primer signo de la emergencia de lo que en los últimos años se conoce como: emprendimiento. $\mathrm{O}$, en otros términos, es el correlato empírico de la traslación de los riesgos económicos del capital a los trabajadores. Desplazamiento que se deriva de la erosión en los mecanismos de legitimación tradicionales del capitalismo en tanto que asunción de riesgos por parte del empresario-empleador o provisión de seguridades y garantías por parte del Estado. Así, los dilemas generados por la anomia endémica del trabajo se responden desde estrategias sociales que se mueven en la búsqueda de lo más estable asociado al funcionariado (que se comprende como último reducto de la modernidad salarial) o, lo más libre asociado al auto-empleo y al emprendimiento (como estrategia individualizada a la desinstitucionalización del trabajo) pasando por un sinfín de estrategias intermedias que llegan a cuestionar ambos extremos ${ }^{3}$.

En este orden de cosas, otras tendencias investigadas han sido aquellas que tienen que ver con las rupturas y el desdibujamiento de dicotomías como la de trabajo-ocio, profesión-afición o trabajo-afectos (Pérez-Agote et al., 2005b). Se detecta una tendencia en la cual habría una cantidad mayor de jóvenes que tratarian de poner en relación trabajo con amistades, con aficiones, con compromisos políticos de diferente orden, etc. Como síntesis, prestar atención a la noción de proyecto (vital) permite a estas investigaciones enunciar una de las claves que sintetiza y explica la super-posición y desbordamientos de las dicotomías modernas señaladas. La noción de proyecto vital condensa el entrecruzamiento de ámbitos anteriormente bien delimitados como el trabajo, las amistades, la pareja, la familia, las capacidades subjetivas, etc., y apunta hacia el imperativo contemporáneo que gira en torno a la formación permanente del yo (De Singly, 2005: 111).

El conjunto de estas investigaciones no solo identifican cambios en los marcos de sentido o en la representación del trabajo, sino que las

\footnotetext{
${ }^{3}$ Estas cuestiones han sido estudiadas en profundidad en posteriores investigaciones como las que siguen: Carbajo, 2015; Santamaría, 2011; Tejerina et al., 2012.
} 
disoluciones de fronteras que van más allá de lo que se venía definiendo como una realidad laboral exclusiva de la juventud. Sí antes los individuos podian pensar y proyectar en el modelo de la estabilidad a través del trabajo, la experiencia en la precariedad de los jóvenes a lo largo de los últimos quince años ha cuestionado definitivamente el modelo subjetivo, el centro y las lógicas de acción modernas. Huelga decir que a día de hoy nos podemos encontrar con segmentos enteros de población -adultaque ha vivido en la precariedad desde su ingreso en el mercado laboral y otros muchos que han experimentado el proceso de precarización del mismo. Asi, lo que en un principio se pudo interpretar como una característica transicional (de lo joven) hoy es constitutiva de muchas posiciones sociales, incluso la del sujeto adulto.

En este sentido, una de las más importantes disoluciones que se identifican en los trabajos dirigidos por Pérez-Agote y que ha sentado las bases para posteriores investigaciones es la que tiene que ver con la dicotomía jovenadulto. Identificar el desdibujamiento de esa frontera ha permitido plantear que la desinstitucionalización y des-ritualización del pasaje de la edad joven a la adulta, su alargamiento, está estrechamente unido con la precarización de lo adulto (Carbajo, 2015), esto es, con la quiebra del individuo moderno a la que apunta en varios de sus trabajos teóricos (Pérez-Agote et al., 2005b: 17; Pérez-Agote, 1996).

\section{IDENTIDADES POST-LABORALES: LA FIGURA DEL SUJETO EMPRENDEDOR}

Los desarrollos sobre las nuevas condiciones sociales que impone la globalización, sumados a otros procesos como el de la financiarización, quedan marcados por una inseguridad endémica y estructural que se imprime en lo laboral. Pero este proceso trasciende ese plano y se termina reflejando en los modos de vida y en los proyectos vitales o biografías que, cada vez más, se constituyen a partir de la incertidumbre, la fragmentación y la fragilización de los vínculos.

Tomando como punto de entrada el actual estado de la cuestión laboral puede decirse que éste convoca a sujetos que asuman riesgos, que tengan gran capacidad de adaptación a un entorno cambiante y que aprovechen las oportunidades que les surgen en el proceso continuo de búsqueda de su desarrollo profesional. Con ello no sólo se disciplinan cuerpos o se extrae fuerza de trabajo al modo moderno (que es lo que se acordaba en el 
contrato de trabajo), sino que destaca cierta insistencia sobre el moldeamiento de la subjetividad que le acompaña (Rose, 1999; Crespo et al., 2009).

Dentro de un llamado general a la formación continua, esta insistencia sobre la subjetividad se convierte en un trabajo sobre uno mismo mediante el cual se busca la adquisición de nuevas capacidades con el objetivo de estar al día y no perder valor respecto a los cambiantes requerimientos del mercado de trabajo. Es también a través de éste tipo de lógicas (la formación continua, la empleabilidad, el multi-empleo, cuasiempleos, etc.) que las identidades laborales contemporáneas ponen en entredicho la operatividad de las categorías analíticas modernas: el rol que venía dado desde el mercado de trabajo se disuelve con otros muchos ámbitos y el sujeto productivo se disgrega. Entonces, resulta pertinente preguntarse: ¿qué tipo de representaciones de individuo pueden resultarnos útiles precisamente cuando los centros principales que las producian -Estado y trabajo, en este caso- cambian de funciones y se difuminan?

Nuestra propuesta se resume en que las disoluciones de fronteras que apuntábamos anteriormente pueden ser condensadas en la figura teóricoanalítica del emprendedor. A grandes rasgos, el emprendimiento, entretejido con otros conceptos como el de empleabilidad o flexiseguridad (Crespo y Serrano, 2011), señala una gramática social en la que la vida adquiere la forma de una empresa (un proyecto vital) en la que todo ámbito de la misma es susceptible de ser gestionado como un capital (patrimonio, amigos, afectos, aficiones, etc.) Aunque el concepto no es nuevo, sí lo es su lugar privilegiado en las políticas de empleo dirigidas no sólo a jóvenes, sino también a otros colectivos como las personas desempleadas, inmigrantes $y$, de forma genérica, a los adultos. Pero especialmente, lo que lo llama la atención a día de hoy es su calado en el imaginario social contemporáneo interpelando a los individuos desde diferentes lugares, no sólo desde lo laboral, a convertirse en sujetos emprendedores ${ }^{4}$.

\footnotetext{
4 Para profundizar en estas cuestiones, véase la literatura académica sobre el emprendimiento: Ginesta, 2013; Kelly, 2013; Lazzarato, 2013; Pfeilstetter, R. 2011. Y valga como muestra anecdótica del calado de la figura del emprendedor, la salida a la venta en el año 2014 de Barbie emprendedora. La conocida muñeca de Mattel que tantos roles ha tenido a lo largo de décadas, representa ahora a una mujer "de su tiempo" y tiene como objetivo "motivar a las chicas para que aprendan este nuevo rol".
} 
Decíamos de la mano de Pérez-Agote que el Estado funcionó durante algún tiempo como la forma de gestión colectiva de los procesos de crisis y anomia. Sin embargo, la emergencia reciente del emprendimiento y la empleabilidad como objetivos prioritarios en políticas y programas institucionales certifica una vez más la idea de que aquellas situaciones y conflictos colectivos que trataba de resolver el Estado se declinan ahora como problemas de voluntad individuales (Keune y Serrano, 2014). Si el Estado y sus mecanismos asistenciales actuales buscan la adaptabilidad de los individuos a una situación anómica y endémica del mundo del trabajo (Serrano et al., 2012), ¿no se descubre con todo esto y una vez más, al Estado -con sus leyes y programas europeos sobre emprendedores y todo su entramado asistencial- como un dispositivo gubernamental del rendimiento económico? ¿No convoca el Mercado, a través del Estado, aquel tipo "empresario de sí mismo" al que apuntaba Foucault en sus cursos del 79 (2007: 249 y ss.)?

Siguiendo a Pérez-Agote, en la modernidad y con la secularización del Estado se genera también la figura de individuo. Se libera éste entonces del Rey, de Dios y en menor medida del Estado y del Mercado (Pérez-Agote, 1996: 28). Si la lógica moderna-disciplinaria todavía funcionaba en base a normas, reglas y coerciones en la que el sujeto producía, la lógica neoliberal actual apela a las nociones de autonomía y de libertad (Martuccelli, 2007) que se constatan claramente en el emprendimiento. Se ha pasado del sujeto productivo al sujeto del rendimiento en la que la noción de proyecto vital revela una actividad ininterrumpida sobre sí (Han, 2012). La asertividad y la positividad a la que alude esta noción de libertad devienen mucho más productivas que el castigo y la negatividad modernas pues la auto-referencialidad y la realización de sí se convierten en el combustible de la acción y la superación del estado anómico. La importancia de este cambio se sitúa en que lo productivo y los objetivos no parecen marcados ya ni por el Estado, ni por el Mercado, sino por la propia subjetividad "liberada ya" de toda determinación heterónoma (Han, 2014). Sin embargo, la tensión en la que se producen o la contradicción en la que se dirimen los individuos contemporáneos gira en torno a un discurso que convoca a aquel sujeto heroico, centrado, auto-sostenido y libre característico de la modernidad en una coyuntura que precisamente los precariza y desestabiliza (Carbajo, 2015).

Si la auto-conciencia del yo es una experiencia generalizada en la modernidad que se agudiza hoy en día (Martuccelli y De Singly, 2012): 
¿tiene recorrido hablar de una mercantilización de la subjetividad en la que la gestión económica del yo resulta clave? O en términos de Alfonso PérezAgote: ¿se puede hablar una forma de neo-protestantismo en el que, articulada respecto a una ética ascética del emprendedor, cada cual se ha convertido en el responsable de su salvación?

\section{Bibliografía}

Carbajo, D., 2015, "Los procesos de precarización de la juventud en la CAPV a través de sus trayectorias residenciales", en Cuadernos Sociológicos Vascos (Vol. 29), Servicio Central de Publicaciones del Gobierno Vasco, Vitoria-Gasteiz.

Crespo, E., Prieto, C., Serrano, A., 2009, Trabajo, subjetividad y ciudadanía: paradojas del empleo en una sociedad en transformación, Centro de Investigaciones Sociológicas y Universidad Complutense de Madrid, Madrid.

Crespo, E., Serrano, A., 2011, "Regulación del trabajo y el gobierno de la subjetividad: La psicologización política del trabajo", en A. Ovejero Bernal (Coord.), Psicología Social Crítica, Biblioteca Nueva, Madrid, pp. 246-263.

De Singly, F., 2005, "Las formas de terminar y no terminar la juventud", en Revista de Estudios de Juventud, n. 71, pp. 109-119.

Foucault, M., 2007, Nacimiento de la biopolítica: curso en el Collège de France (1978-1979), Fondo de Cultura Económica, México.

Ginesta, V., 2013, "Apología del emprendedor: análisis crítico del discurso sobre el interés propio", en Oxímora, Revista Internacional de Ética y Política, 3, pp. 56-74.

Han, B.C., 2012, La sociedad del cansancio, Herder, Barcelona.

Han, B.C., 2014, Psicopolítica. Neoliberalismo y nuevas técnicas de poder, Herder, Barcelona.

Kelly, P., 2013, The Self as Enterprise: Foucault and the Spirit of 21st Century Capitalism, Ashgate/Grower, Surrey.

Keune, M., Serrano, A., (Eds.), 2014, Deconstructing Flexicurity and Developing Alternative Approaches, Routledge, London.

Martuccelli, D., 2007, Gramáticas del individuo, Losada, Buenos Aires.

Martuccelli, D., De Singly, F., 2012, Las sociologias del individuo, LOM, Santiago de Chile. 
Lazzarato M., 2013, La fábrica del hombre endeudado. Ensayo sobre la condición neoliberal, Amorrortu, Buenos Aires.

Pérez-Agote, A., 1996, "La sociedad se difumina, el individuo se disgrega", en Pérez-Agote, A. y Sánchez De La Yncera, I., (Eds.), Complejidad y Teoría Social, CIS, Madrid, pp. 11-32.

Pérez-Agote, A., 1999, "Globalización, crisis del Estado y anomía. La teoría social visita Europa" en R. Ramos Torre y F. García Selgas (Eds.), Globalización, riesgo y reflexividad. Tres temas de la teoría social contemporánea, CIS, Madrid, pp. 57-72.

Pérez-Agote, A., 2000, "La crisis de la homogeneidad cultural", en VV. AA, El bienestar en la cultura. Estudios de la Facultad de Ciencias Sociales y de la Comunicación en homenaje al Profesor Iñaki Dominguez Vázquez, UPV/EHU, Bilbao, pp. 439-459.

Pérez-Agote, A., 2002, "¿Morir de éxito?: Fin de la modernidad y triunfo de lo social", en J.M. García Blanco y P. Navarro P. (Eds.), ¿Más allá de la modernidad?: las dimensiones de la información, la comunicación y sus nuevas tecnologías, CIS, Madrid, pp. 505-526.

Pérez-Agote, A., Santamaría, E., 2008, Emancipación y precariedad en la juventud vasca. Entre la anomia funcional y el cambio cultural, Servicio Central de Publicaciones del Gobierno Vasco, VitoriaGasteiz.

Pérez-Agote, A., Tejerina, B., Santamaría, E., 2001, “La inserción laboral de los jóvenes en Bizkaia", en Cuadernos de empleo, Gazte Lanbidean BBK Fundazioa, Bilbao.

Pérez-Agote, A., Tejerina, B., Santamaría, E., 2005a, "Transformaciones y tendencias de la cultura del trabajo en Bizkaia. Enfoque cuantitativo", en Cuadernos de empleo, Gazte Lanbidean BBK Fundazioa, Bilbao.

Pérez-Agote, A., Tejerina, B., Gatti, G., Martinez De Albeniz, I., Seguel, A.G., Cavia, B., Santamaría, E., 2005b, "Hacia una nueva cultura de la identidad y la política", en Tendencias de la juventud vasca (Vol. 1), Servicio Central de Publicaciones del Gobierno Vasco, VitoriaGasteiz.

Pfeilstetter, R., 2011, "El emprendedor. Una reflexión crítica sobre usos y significados actuales de un concepto", en Gazeta de Antropología, 27 (1), pp. 1-11.

Rose, N., 1999, Governing the soul the shaping of the private self, Free Association Books, London. 
Santamaría, E., 2011, Trayectorias laborales en los márgenes del empleo: políticas, subjetividades y experiencias de jóvenes en la precariedad laboral, Servicio Central de Publicaciones del Gobierno Vasco, Vitoria-Gasteiz.

Serrano, A., Fernández Rodríguez, C.J., Artiaga, A., 2012, "Ingenierías de la subjetividad: el caso de la orientación para el empleo", en Reis: Revista española de investigaciones sociológicas, n. 138, pp. 41-62.

Tejerina, B., Cavia, B., Santamaría, E., Carbajo, D., 2012, Precariedad vital y juventud vasca. Condiciones sociales y estrategias biográficas para llevar una vida normal, Servicio Central de Publicaciones del Gobierno Vasco, Vitoria-Gasteiz. 